\title{
Antifungal Mechanism of Vip3Aa, a Vegetative Insecticidal Protein, against Pathogenic Fungal Strains
}

\author{
Seong-Cheol Park ${ }^{1}$, Jin-Young Kim ${ }^{1}$, Jong-Kook Lee ${ }^{1}$, Hye Song Lim ${ }^{2}$, Hyosuk Son ${ }^{1,3}$, Su-Hyang Yoo ${ }^{2}$, \\ Seong-Eun Mun ${ }^{4}$, Mi-Kyeong Jang ${ }^{1, *}$ and Jung Ro Lee ${ }^{2, * \mathbb{D}}$ \\ 1 Department of Chemical Engineering, Sunchon National University, Suncheon 57922, Korea; \\ schpark9@gnu.ac.kr (S.-C.P.); jyfrog@hanmail.net (J.-Y.K.); seal9669@naver.com (J.-K.L.); \\ skitten74@mabik.re.kr (H.S.) \\ 2 LMO Research Team, National Institute of Ecology, 1210 Geumgang-ro, Maseo-myeon, \\ Seocheon-gun 33657, Korea; hslim0826@nie.re.kr (H.S.L.); hyang77@nie.re.kr (S.-H.Y.) \\ 3 National Marine Biodiversity Institute of Korea, 101-75 Jangsan-ro, Janghang-eup, Seocheon-gun 33662, Korea \\ 4 Department of Biological Science, College of Natural Science, Wonkwang University, Iksan 54538, Korea; \\ showmse@nate.com \\ * Correspondence: jmk8856@sunchon.ac.kr (M.-K.J.); leejr73@nie.re.kr (J.R.L.); Tel.: +82-62-750-3567 (M.-K.J.); \\ $+82-41-950-5820$ (J.R.L.)
}

\section{check for} updates

Citation: Park, S.-C.; Kim, J.-Y.; Lee, J.-K.; Lim, H.S.; Son, H.; Yoo, S.-H.; Mun, S.-E.; Jang, M.-K.; Lee, J.R. Antifungal Mechanism of Vip3Aa, a Vegetative Insecticidal Protein, against Pathogenic Fungal Strains. Antibiotics 2021, 10, 1558. https://doi.org/10.3390/ antibiotics 10121558

Academic Editors: Sumant Puri and Gill Diamond

Received: 3 November 2021

Accepted: 17 December 2021

Published: 20 December 2021

Publisher's Note: MDPI stays neutral with regard to jurisdictional claims in published maps and institutional affiliations.

Copyright: (c) 2021 by the authors. Licensee MDPI, Basel, Switzerland. This article is an open access article distributed under the terms and conditions of the Creative Commons Attribution (CC BY) license (https:/ / creativecommons.org/licenses/by/ $4.0 /)$.

\begin{abstract}
Discovering new antifungal agents is difficult, since, unlike bacteria, mammalian and fungal cells are both eukaryotes. An efficient strategy is to consider new antimicrobial proteins that have variety of action mechanisms. In this study, a cDNA encoding Bacillus thuringiensis Vip3Aa protein, a vegetative insecticidal protein, was obtained at the vegetative growth stage; its antifungal activity and mechanism were evaluated using a bacterially expressed recombinant Vip3Aa protein. The Vip3Aa protein demonstrated various concentration- and time-dependent antifungal activities, with inhibitory concentrations against yeast and filamentous fungi ranging from 62.5 to $125 \mu \mathrm{g} / \mathrm{mL}$ and 250 to $500 \mu \mathrm{g} / \mathrm{mL}$, respectively. The uptake of propidium iodide and cellular distributions of rhodamine-labeled Vip3Aa into fungal cells indicate that its growth inhibition mechanism involves its penetration within cells and subsequent intracellular damage. Furthermore, we discovered that the death of Candida albicans cells was caused by the induction of apoptosis via the generation of mitochondrial reactive oxygen species and binding to nucleic acids. The presence of significantly enlarged Vip3Aa-treated fungal cells indicates that this protein causes intracellular damage. Our findings suggest that Vip3Aa protein has potential applications in the development of natural antimicrobial agents.
\end{abstract}

Keywords: antifungal activity; Vip3Aa protein; reactive oxygen species; Bacillus thuringiensis

\section{Introduction}

Bacillus thuringiensis is the most widely used biological insecticide for controlling insect pests, primarily Lepidoptera and Coleoptera species [1]. Among the insecticidal proteins secreted by $B$. thuringiensis, the parasporal inclusion crystal (Cry) toxins are the most wellknown and widely used. Cry toxins accumulate during sporulation in the B. thuringiensis strain, resulting in a crystalline inclusion with a variety of morphologies. When pests consume Cry toxins, the alkaline digestive tract of the insects denatures the insoluble crystals, making them soluble and thus susceptible to digestion by proteases found in the pest gut, which releases the toxin from the crystal [2]. Following this, the Cry toxin penetrates the cell membrane of the pest digestive tract, paralyzing the gut and forming a pore. The pest eventually stops eating and starves to death $[2,3]$.

Vegetative insecticidal protein (Vip) is another type of insecticidal protein produced by B. thuringiensis and B. cereus [3-7]. Vip proteins are released during vegetative growth and have no resemblance to Cry toxins. Until recently, the Vip protein family was divided into 
four categories: Vip1, Vip2, Vip3, and Vip4 [8]. Vip1 and Vip2 proteins are the two parts of a binary toxin, which is toxic to coleopterans. Vip1Aa1 and Vip2Aa1 have high anti-corn rootworm activity [6,9]. The currently known insecticidal molecular mechanism of Vip1Ac toxins begins with the larva ingesting the toxin. The monomer of Vip1Ac activated by the protease in the larva's midgut forms oligomers containing seven Vip1 molecules [10]. These oligomers bind to specific receptors on the mid-gut border membrane, where the Vip1Ac toxin is then inserted.

Vip3 proteins have a diverse host range, which includes a number of major lepidopteran pests [3-9]. Vip3A proteins must be cleaved by proteases prior to the recognition of specific $80 \mathrm{kDa}$ and $100 \mathrm{kDa}$ membrane proteins, different from those perceived by Cry toxins at the surface of the mid-gut epithelium [11]. Among the Vip3 family, Warren's study identified Vip3Aa $(89 \mathrm{kDa})$, which has high toxicity to Agrotis ipsilon and other lepidopteran larvae [6]. Vip3A protein has recently been discovered to be a pore-forming protein capable of forming stable ion channels in the membrane [11]. The $\mathrm{pH}$ of the solution is one of the factors that may influence the insecticidal activity of the Vip3Aa protein. The $\mathrm{pH}$ of lepidopteran midgut lumen ranges from 8.0 to 12.0 [12,13]. Proteolysis by mid-gut proteases is most effective at $\mathrm{pH}$ 10.0-12.0. An alkaline condition is required for the Vip3Aa toxin to be converted to its toxic form [12]. However, the effect of $\mathrm{pH}$ on the functional properties of Vip3Aa is yet to be determined.

The positively charged hydrophobic region at the N-terminus of Vip3 proteins suggests that this region is important for protein structure and insecticidal activity [4,14-17]. Furthermore, the last amino acid at the C-terminus is known to play a role in Vip3 protein activity and safety. Most Vip3 proteins have a carbohydrate binding motif, which spans from position 536 to a position near amino acid 652 at the C-terminus [14,15]. Furthermore, recent research has shown that both 19-22 kDa (N-terminus region) and 62-66 kDa (C-terminus region) fragments are required for the stability and specificity of Vip3A toxins [18]. A $340 \mathrm{kDa}$ homo-tetramer composed of the 19-22 kDa and 62-66 kDa fragments of Vip3A was identified after digestion with trypsin or insect mid-gut proteases $[19,20]$. Quan Y et al., discovered that Vip3Af mutants form structurally diverse oligomers, and Ensi Shao et al. discovered that protein oligomers formed of $19 \mathrm{kDa}$ and $65 \mathrm{kDa}$ fragments of Vip3Aa were critical for insecticidal toxicity [21,22].

While studying the insecticidal activity of the Vip3Aa protein, we developed an interest in the characteristics and novel functions of the Vip3Aa protein, which are similar to those of common antimicrobial peptides (AMPs) [22]. In most organisms, AMPs are involved in the innate host defense system as a primary barrier against infection. Research on AMPs has focused on their abundance in nature, their mechanisms, and their roles in immune systems. The aim of this study was to confirm the $\mathrm{pH}$-dependent antimicrobial activity potential of Vip3Aa protein. As a result, the Vip3A protein demonstrated antifungal activity only at a specific $\mathrm{pH}$. In addition to its insecticidal activity, use of Vip3Aa as a potential antibiotic as part of a biological control strategy was also confirmed.

\section{Results}

\subsection{Antifungal Activity of Vip3Aa Protein against Pathogenic Fungi}

To determine the antimicrobial activity of B. thuringiensis Vip3Aa, we expressed recombinant Vip3Aa protein in Escherichia coli. The full-length cDNA of Vip3Aa was cloned into the pET28a vector and expressed in E. coli BL21(DE3). To confirm the structural orientation and purity of the isolated recombinant protein, size-exclusion chromatography (SEC) and 10\% sodium dodecyl sulphate-polyacrylamide gel electrophoresis (SDS-PAGE) analyses were performed (Figure 1A,B). Analysis of SEC purified fractions revealed that the native molecular weight of Vip3Aa was $669 \mathrm{kDa}$, which may have an oligomeric structure of at least an octamer or more or an irregular highly aggregated structure (Figure 1A). It is known that the C-terminus of Vip3A proteins are cleaved by midgut proteases to produce a 62-66 kDa protease-resistant toxic core [8,23]. Recently, an approximately $340 \mathrm{kDa}$ homotetramer structure has been identified from Vip3A digested by trypsin or insect midgut 
proteases $[19,21,22,24]$. However, the native structure of Pro-Vip3Aa has not been clearly identified. Negative staining on a transmission electron microscope (TEM) revealed that the forms of Vip3Aa proteins fractionated on SEC represent a regular oligomer (Figure 1C). These physicochemical analyses show that recombinant Vip3Aa was obtained in a purified form, and this protein has oligomeric structures in its native state. In addition, $\mathrm{pH}$ is a very important factor in the insecticidal function of Vip3Aa. Therefore, the $\mathrm{pH}$-dependent structural changes of Vip3Aa protein were confirmed using TEM. In assays from $\mathrm{pH} 5.5$ to 9.5, the proteins of Vip3Aa formed significant self-aggregated complexes, which increased in size by acidification (Figure 1D). We visually confirmed abnormal precipitates in the protein solution under weakly acidic conditions. This may be the initial state of protein crystallization, but it is an important factor in the antifungal activity of the Vip3Aa protein proposed in this study.

A

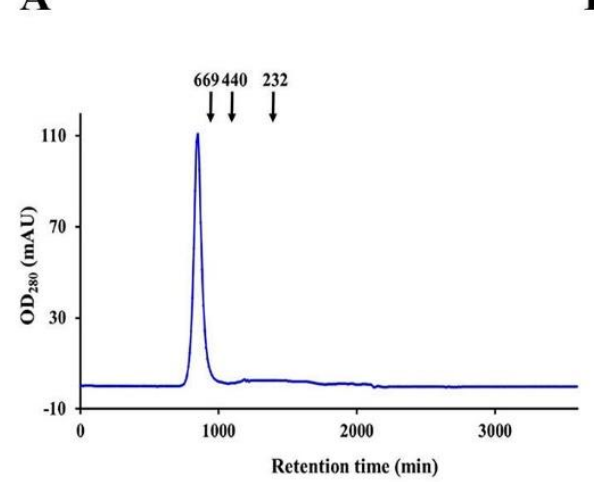

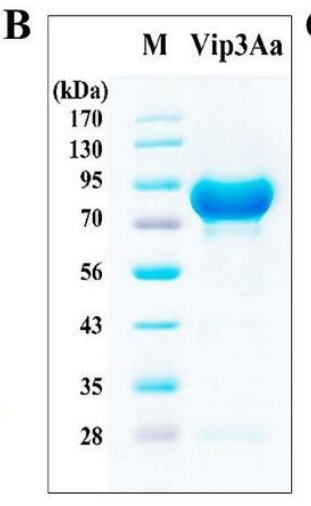
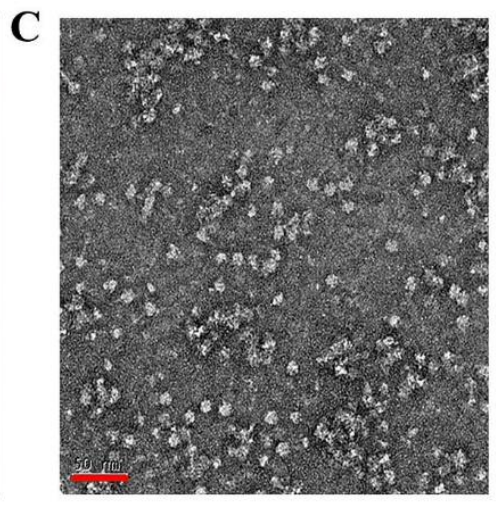

D

$$
\text { pH } 5.5
$$

pH 6.5

pH 7.5

pH 8.5

pH 9.5

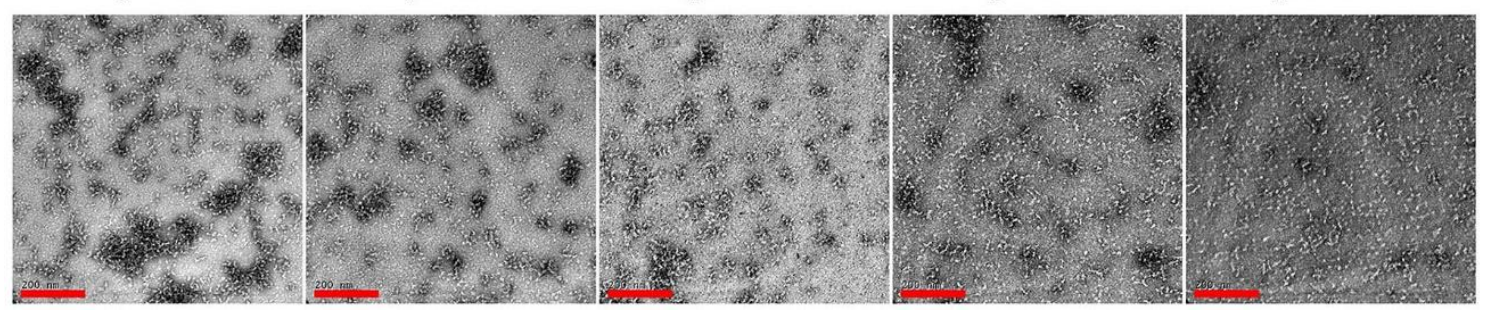

Figure 1. Purification and characterization of recombinant Vip3Aa protein. (A) Recombinant Vip3Aa protein was purified on SEC. The short vertical lines indicate standard markers that were calibrated with bovine thyroglobulin (669 kDa), ferritin (440 kDa), and bovine catalase (232 kDa). (B) The purified recombinant Vip3Aa from E. coli was resolved by 10\% SDS-PAGE. (C) Oligomeric forms of Vip3Aa protein fractionated from SEC ( $\mathrm{pH}$ 9.5) were observed under TEM. Bar presents $50 \mathrm{~nm}$. (D) TEM analysis of self-aggregation of Vip3Aa protein in a pH-dependent manner. Bars present $200 \mathrm{~nm}$.

In this study, the antifungal activity of Vip3Aa was investigated by determining the inhibitory concentration (IC) against seven fungi species. Melittin, which is derived from honeybee venom, is well known as a natural peptide with high cytotoxicity as well as excellent antimicrobial activity via membranolytic action $[25,26]$. Because it exists as an ordered self-aggregate in aqueous solution and forms toroidal pores in fungal cells [25,26], we used it as a comparative control. Melittin inhibited the growth of tested fungal cells at $\mathrm{IC}_{50}$ values ranged from 11 to $88 \mu \mathrm{M}$, while $\mathrm{IC}_{50}$ values of Vip3Aa ranged from 0.7 to $5.6 \mu \mathrm{M}$ (Table 1). The MIC values determined after $24 \mathrm{~h}$ or $48 \mathrm{~h}$ treatments of Vip3Aa were 1.4 to $2.8 \mu \mathrm{M}$ against yeast cells and 5.6 to $11.1 \mu \mathrm{M}$ against mold cells. The molecular weight of the monomeric melittin is about $2.8 \mathrm{kDa}$, and the recombinant Vip3Aa is about $90 \mathrm{kDa}$, which is a 32-fold difference. Furthermore, melittin exists as a tetramer and Vip3Aa forms an oligomer as at least an octamer in aqueous solution. When compared with molar concentration, it indicates that the antifungal activity of Vip3Aa is more potent than that of 
melittin. The antifungal activity of both proteins was better against yeast cells than mold cells within the tested fungal cells.

Table 1. Antifungal activity of Vip3Aa against fungal cells.

\begin{tabular}{|c|c|c|c|c|}
\hline \multirow{3}{*}{ Fungal Strains } & \multicolumn{2}{|c|}{ Vip3Aa } & \multicolumn{2}{|c|}{ Melittin } \\
\hline & \multicolumn{4}{|c|}{$\mu \mathbf{M}(\mu \mathrm{g} / \mathrm{mL})$} \\
\hline & $\mathrm{IC}_{50}$ & MIC & $\mathrm{IC}_{50}$ & MIC \\
\hline \multicolumn{5}{|c|}{ Yeast } \\
\hline C. albicans & $0.7(62.5)$ & $1.4(125)$ & $11(31.3)$ & $22(62.5)$ \\
\hline C. krusei & $0.7(62.5)$ & $1.4(125)$ & $11(31.3)$ & $22(62.5)$ \\
\hline C. parapsilosis & $1.4(125)$ & $2.8(250)$ & $22(62.5)$ & $44(125)$ \\
\hline C. tropicalis & $1.4(125)$ & $2.8(250)$ & $22(62.5)$ & $44(125)$ \\
\hline \multicolumn{5}{|c|}{ Molds } \\
\hline Colletotrichum gloeosporioides & $5.6(500)$ & $11.1(1000)$ & $88(250)$ & $176(500)$ \\
\hline F. graminearum & $5.6(500)$ & $11.1(1000)$ & $88(250)$ & $176(500)$ \\
\hline F. solani & $2.8(250)$ & $5.6(500)$ & $88(250)$ & $88(250)$ \\
\hline
\end{tabular}

To be used as an antifungal agent, all compounds or materials have to possess nontoxic character. We examined the hemolytic and cytotoxic effects on rat erythrocytes and human $\mathrm{HaCaT}$ cells, respectively. As shown in Figure 2A, melittin exhibited 91.7\% hemolysis even at a low concentration of $3.125 \mu \mathrm{g} / \mathrm{mL}$, whereas Vip3Aa and BSA achieved $6.3 \%$ and $3.9 \%$ hemolysis, respectively, at a high concentration of $500 \mu \mathrm{g} / \mathrm{mL}$. On the other hand, in $\mathrm{HaCaT}$ cells, human keratinocytes, melittin at $6.25 \mu \mathrm{g} / \mathrm{mL}$ resulted in $1.9 \%$ cell survival, but the cells treated with $500 \mu \mathrm{g} / \mathrm{mL}$ of Vip3Aa and BSA survived to $90.7 \%$ and $95.4 \%$, respectively (Figure 2B). Although Vip3Aa causes extensive damage in the midgut of insects (a eukaryote) by forming transmembrane pores, these findings suggest that it causes cell-selective death at least in fungal and human cells. Recently, Yang et al., reported that Cry protein, an insecticidal protein that is similar to Vip3Aa, can be safely used as a peptide delivery carrier in vivo [27].
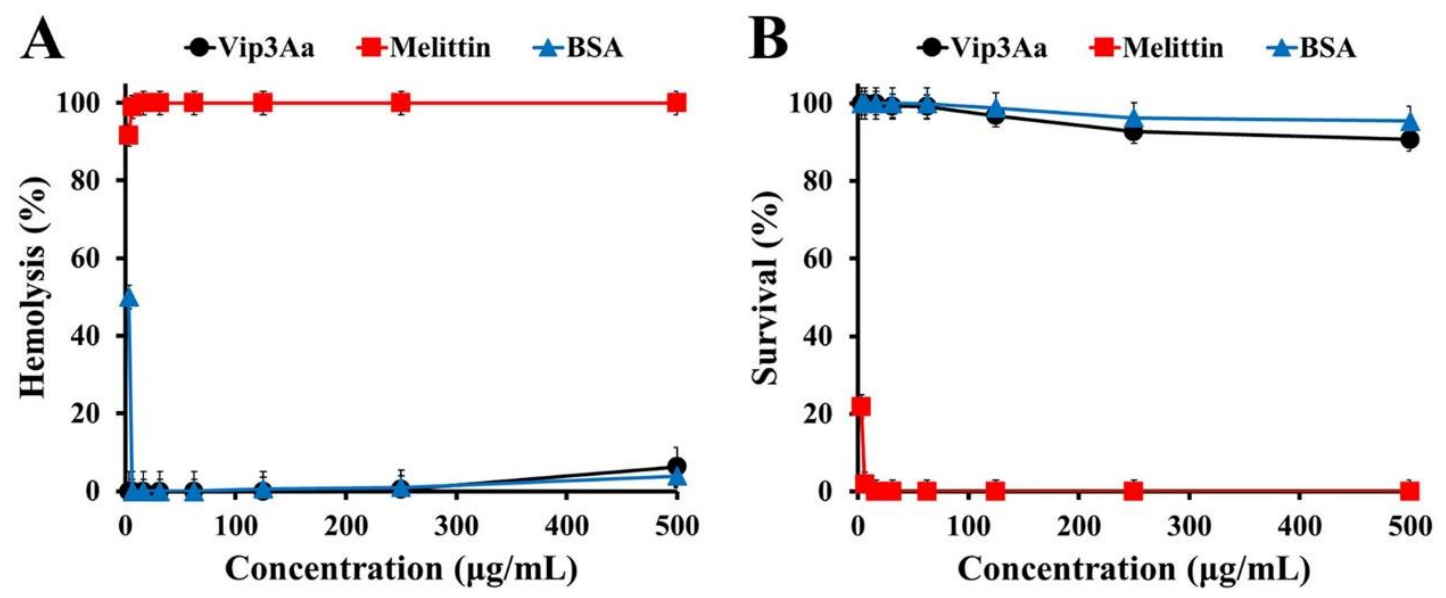

Figure 2. Hemolysis and cytotoxicity of Vip3Aa against rat erythrocytes (A) and HaCaT cells (B). (A) After $1 \mathrm{~h}$ incubation in the presence of proteins at the represented concentration, the absorbance of hemoglobin released from rat erythrocytes was measured at $414 \mathrm{~nm}$. (B) After $24 \mathrm{~h}$ incubation of proteins in HaCaT cells, cell survival was assayed by activated-XTT solution.

\subsection{Molecular Mechanism of the Vip3Aa Protein in Fungal Cells}

As shown in Table 1, Vip3Aa was found to possess antifungal properties against yeast and filamentous fungi. Before the mechanism study, the visible growth patterns or morphological changes of $C$. albicans cells according to the concentration and incubation time of Vip3Aa were observed under a microscope (Figure 3A,B). As shown in Figure 3A, 
depending on the treated Vip3Aa concentration, cell proliferation was significantly inhibited, compared to the control. The morphologies of Vip3Aa-treated C. albicans cells were ring-shaped (Figure $3 \mathrm{~A}, \mathrm{~B}(2-4)$ ), despite the fact that their normal phenotypes are oval (Figure $3 \mathrm{~A}, \mathrm{~B}(1)$ ). Since the number and size of ring-shaped cells vary upon the treating Vip3Aa concentration, the antifungal effects and cell morphologies of recombinant Vip3Aa were studied in C. albicans cells for a $4-48 \mathrm{~h}$ incubation period. As a result, the size of ring-shaped cells increased for $24 \mathrm{~h}$, but most of the cells shrank, and cell debris increased after $48 \mathrm{~h}$. This can occur by inhibiting cell wall biosynthesis or cell division or by inducing apoptosis (Figure 3B). Yeast cells generally produce daughter cells by budding and continued cell proliferation. Under normal conditions, chain-type budding occurs rarely. However, chain-type budding was observed in most of the Vip3Aa-treated C. albicans cells at low concentration or for short incubation time. Vip3Aa may act on C. albicans cells via intracellular damage rather than causing direct membrane damage.

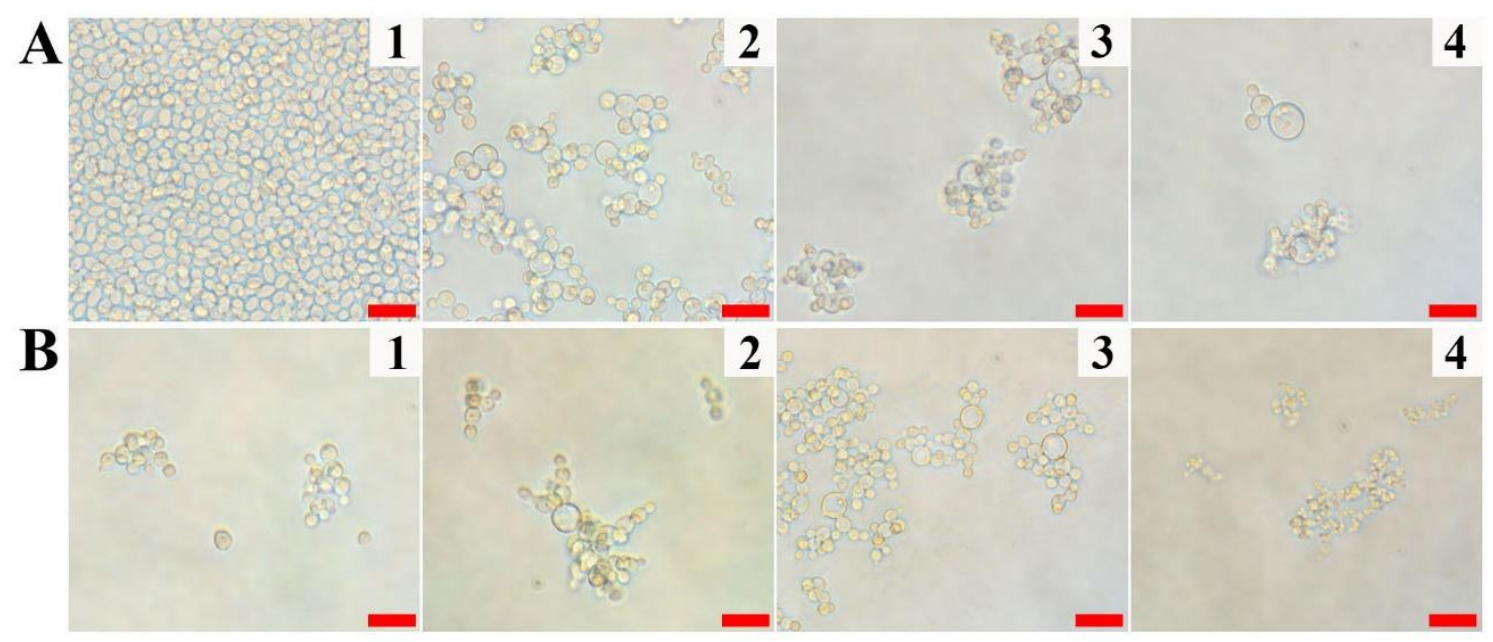

Figure 3. Concentration and time-dependent antifungal effects of recombinant Vip3Aa in C. albicans cells. (A) Concentrationdependent inhibition of fungal growth at $12 \mathrm{~h}$ incubation. (1): Control, (2): $31.3 \mu \mathrm{g} / \mathrm{mL}$ Vip3Aa, (3): $62.5 \mu \mathrm{g} / \mathrm{mL}$ Vip3Aa, (4): $125 \mu \mathrm{g} / \mathrm{mL}$ Vip3Aa. (B) Time-dependent inhibition of fungal growth by $62.5 \mu \mathrm{g} / \mathrm{mL}$ Vip3Aa. (1): $4 \mathrm{~h},(2): 12 \mathrm{~h}$, (3): $24 \mathrm{~h}$, (4): $48 \mathrm{~h}$. Bars represent $20 \mu \mathrm{m}$.

We propose that the antifungal activity of the protein may be due to its structural alternation from a non-aggregated quaternary structure to the aggregated form via changes in intracellular $\mathrm{pH}$ environment values of fungal cells.

\subsection{Intracellular Localization and Molecular Mechanism of Vip3Aa in Fungal Cells}

To investigate the cell affinity of Vip3Aa, rhodamine was conjugated to amine group proteins. Due to their strong hydrophobicity, fluorescent dyes participate in unwanted side reactions by completely different mechanisms when treated with cells. Hence, we mixed rhodamine-labeled protein and rhodamine-free protein in a weight ratio of 1:9, and $C$. albicans cells were treated with the mixtures at $\mathrm{IC}_{50}$. As shown in Figure $4 \mathrm{~A}$, cells with melittin showed a fluorescence shift of $89.45 \%$ after 30 min of treatment, but Vip3Aa induced a shift of $6.61,20.52$, and $77.18 \%$ after 4,6 , and $12 \mathrm{~h}$, respectively. The binding affinity of proteins to cells and their uptake capacity into cells cannot be determined by flow cytometry alone. Therefore, the cellular distribution of Vip3Aa was visualized using a confocal laser scanning microscope (CLSM) (Figure 4B). Cellular red fluorescence was strong after $6 \mathrm{~h}$ incubation, but the intracellular accumulation of rhodamine-labeled Vip3Aa increased in all treated cells after $12 \mathrm{~h}$ of incubation. 


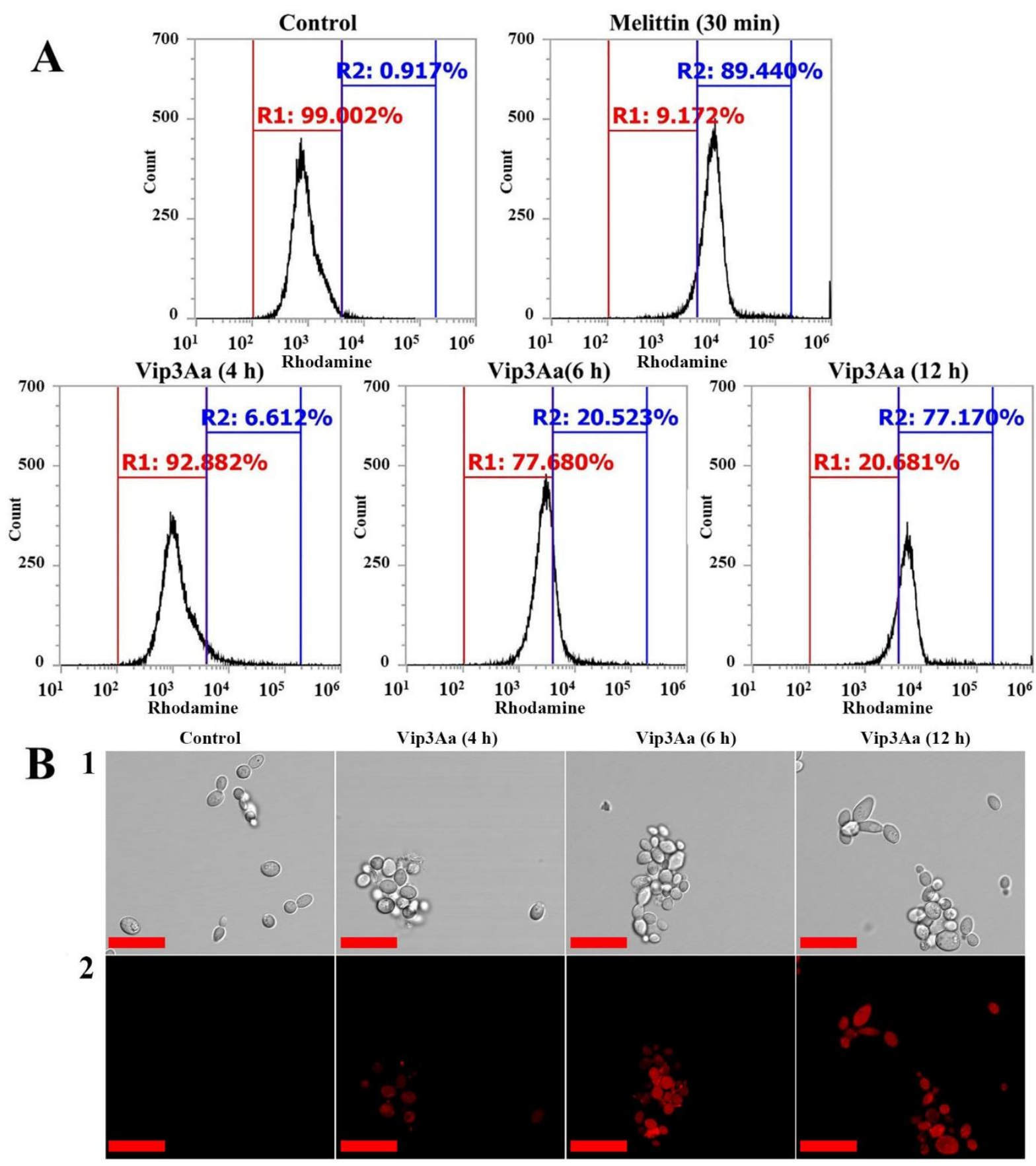

Figure 4. Cellular binding and uptake of Vip3Aa in C. albicans cells. Rhodamine-labeled melittin and Vip3Aa were incubated for the given time points at the $\mathrm{IC}_{50}$ concentration, and the treated cells were analyzed using (A) flow cytometry and (B) confocal laser scanning microscope (CLSM). Bar is $20 \mu \mathrm{m}$. 1: DIC, 2: Rhodamine.

To determine the cellular localization of proteins in fungal cells, C. albicans cells were treated with rhodamine-labeled melittin and Vip3Aa and observed using a CLSM. On the surface of $C$. albicans, the fluorescence of rhodamine-labeled melittin was observed. In contrast, rhodamine-labeled Vip3Aa fluoresced in the cytosol of C. albicans (Figure 5A). These findings imply that Vip3Aa has a different mechanism of action from melittin. A propidium iodide (PI) uptake assay was performed using a flow cytometer to confirm the results obtained (Figure $5 \mathrm{~B}$ ). Because PI is an impermeable dye, it can be penetrated into only cells with membrane damage, and it emits red fluorescence by binding to nucleic acids. Melittin is well known for disrupting fungal membranes by forming pores. Thus, melittin exhibited a rapid PI uptake of 74.7\% after incubating for $30 \mathrm{~min}$. However, Vip3Aa-treated 
cells did not show significant PI uptake even after incubation for $240 \mathrm{~min}$. Thus, we propose that the Vip3Aa protein has potent antifungal activity without membranolysis.

A
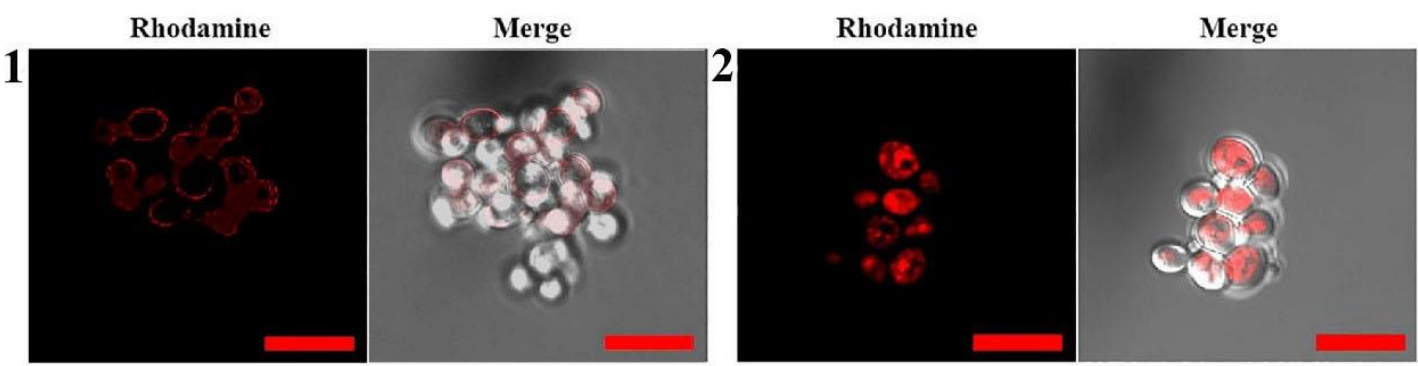

$\underset{100}{\mathbf{B}}$
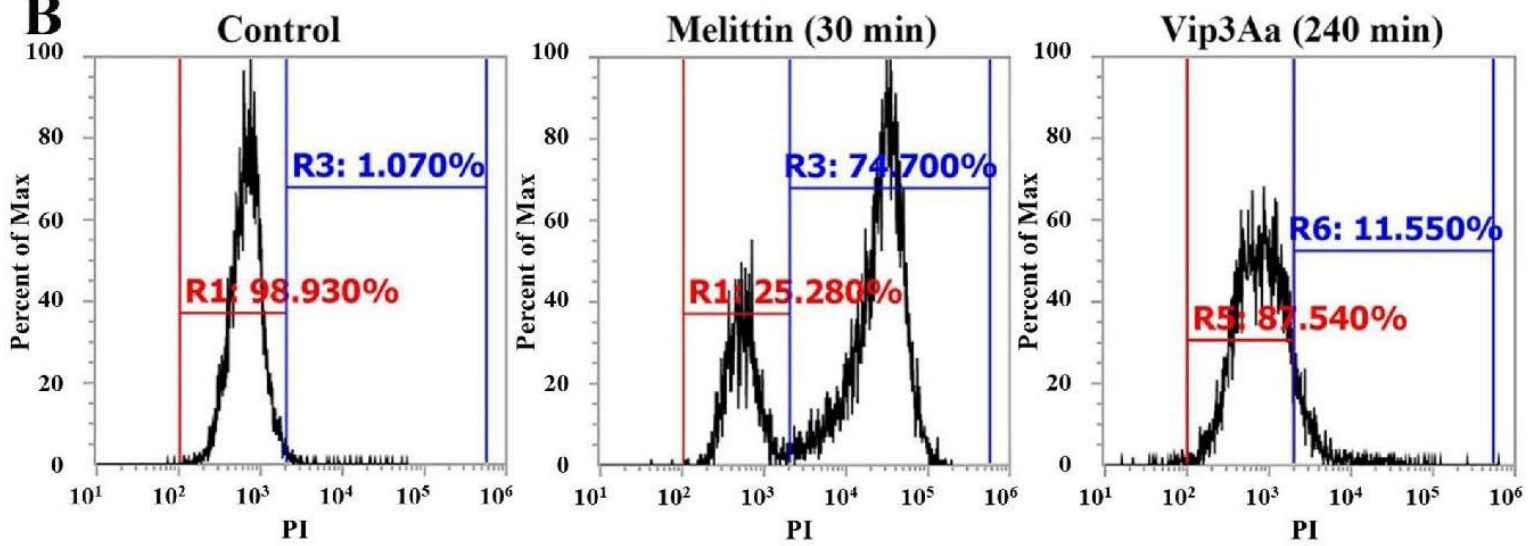

Figure 5. Cellular distributions of Vip3Aa in C. albicans cells. (A) After incubating C. albicans cells with rhodamine-labeled melittin (1) for $1 \mathrm{~h}$ and Vip3Aa (2) for $12 \mathrm{~h}$, the fungal cells were washed and examined using a CSLM. Bar is $10 \mu \mathrm{m}$. (B) Flow cytometry analysis was used to evaluate intracellular uptake of PI in C. albicans cells after incubation with melittin or Vip3Aa.

\subsection{Intracellular ROS Production and Apoptosis Induction by Vip3Aa}

The mechanisms of action of numerous AMPs are broadly classified into the following two categories: membranolytic actions such as membrane potential changes and the formation of pores in the cell membrane; and intracellular inhibiting actions such as interfering gene expression, inhibiting enzyme activity, generating reactive oxygen species (ROS), and inducing osmotic pressure [28,29]. There is growing evidence of AMPs inducing cell death by stimulating the production of ROS [30-34]. Although fungal cells repeat the cycle of generating and eliminating intracellular ROS during metabolic pathways, the high levels of ROS damage intracellular lipids, proteins, DNA, organelles, and cell walls $[35,36]$. To investigate the effects of Vip3Aa on mitochondrial ROS production in C. albicans, mitochondrial superoxide (MitoSOX) Red, a selective mitochondrial fluorescence probe, was monitored using fluorescence microscopy and flow cytometry (Figure 6A). In flow cytometry analysis, cells treated with Vip3Aa for $12 \mathrm{~h}$ showed a 7.6\% increase in MitoSOX production compared to the control, but there are many cells emitting red fluorescence under a fluorescence microscope. This difference is due to excessive cell aggregation and enlarged cell size by the incubation time of $12 \mathrm{~h}$, as shown in cell morphological alterations in Figure 3. To determine whether apoptosis is involved in cell death, the C. albicans cells treated with Vip3Aa were observed using FITC Annexin-V (specifically binding to externalized phospatidylserine) and PI (cell membrane integrity) co-staining method (Figure 6B). Cells treated with Vip3Aa showed early apoptosis (2-12\%), late apoptosis (3-32\%), and necrosis $(2-4 \%)$. With an increase in the concentration of Vip3Aa, apoptosis significantly increased instead of necrosis. This implies that Vip3Aa not only prevents cell proliferation of $C$. albicans but is also involved in cell death. 


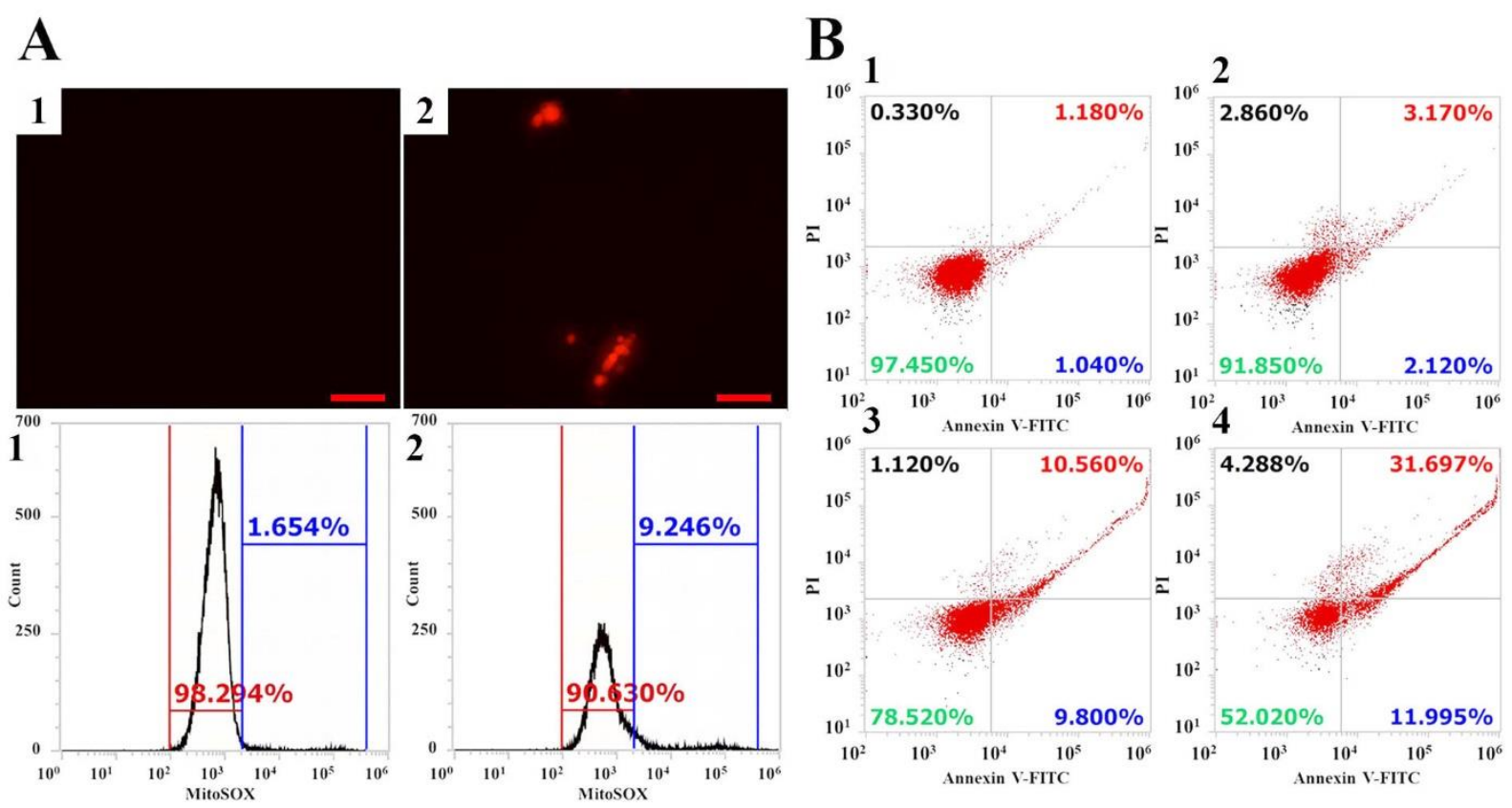

Figure 6. Generation of mitochondrial superoxide (MitoSOX) and induction of apoptosis by Vip3Aa. (A) After incubation of Vip3Aa, MitoSOX probe was added in the cells and analyzed by fluorescence microscopy and flow cytometry. (1): control, (2): Vip3Aa $(62.5 \mu \mathrm{g} / \mathrm{mL})$. Bar is $50 \mu \mathrm{m}$. (B) Vip3Aa proteins were incubated with C. albicans cells for $12 \mathrm{~h}$, and the cells were stained with PI/FITC Annexin-V and analyzed using flow cytometry. (1): Control, (2): $31.3 \mu \mathrm{g} / \mathrm{mL}$ of Vip3Aa, (3): $62.5 \mu \mathrm{g} / \mathrm{mL}$ of Vip3Aa, (4): $125 \mu \mathrm{g} / \mathrm{mL}$ of Vip3Aa.

\subsection{Morphological Alterations Caused by Vip3Aa in Fungal Cells}

Scanning electron microscope (SEM) was used to examine the morphological changes in C. albicans cells in the presence of melittin and Vip3Aa. When compared to untreated cells, melittin-treated cells were wrinkled and had irregular-sized holes in the cell surface after $4 \mathrm{~h}$ of incubation (Figure 7A,B). Cells treated with Vip3Aa at the $\mathrm{IC}_{50}$ level exhibited severely wrinkled cell surfaces and enlarged cell size (Figure 7C-F). The sizes of cells incubated with the Vip3Aa protein were larger than those of control cells, indicating that swelling in the presence of the Vip3Aa protein increased cell size via formation of a single zygote by fusion of yeast cells. The cells incubated with Vip3Aa for $24 \mathrm{~h}$ showed a cracked cell wall with surface roughness and numerous scars.

C. albicans undergoes hyphal growth when it causes pathogenesis, but it continuously produces daughter cells by budding under normal growth conditions. The most important factor in the antifungal mechanism of Vip3Aa is its structural variations with change in environmental $\mathrm{pH}$. During the growth of $C$. albicans cells, the intracellular $\mathrm{pH}$ varies between 6 and 7, owing to glucose metabolism. Penetrated Vip3Aa can be aggregated in acidic cytosol. These can eventually interfere with factors involved in cell division (formation of short cell chains) and induce mitochondrial ROS (cell swelling). In the early stage of Vip3Aa treatment, it inhibits the growth of fungal cells via fungistatic action; however, with time, it develops a fungicidal action that causes cell death via apoptosis. 


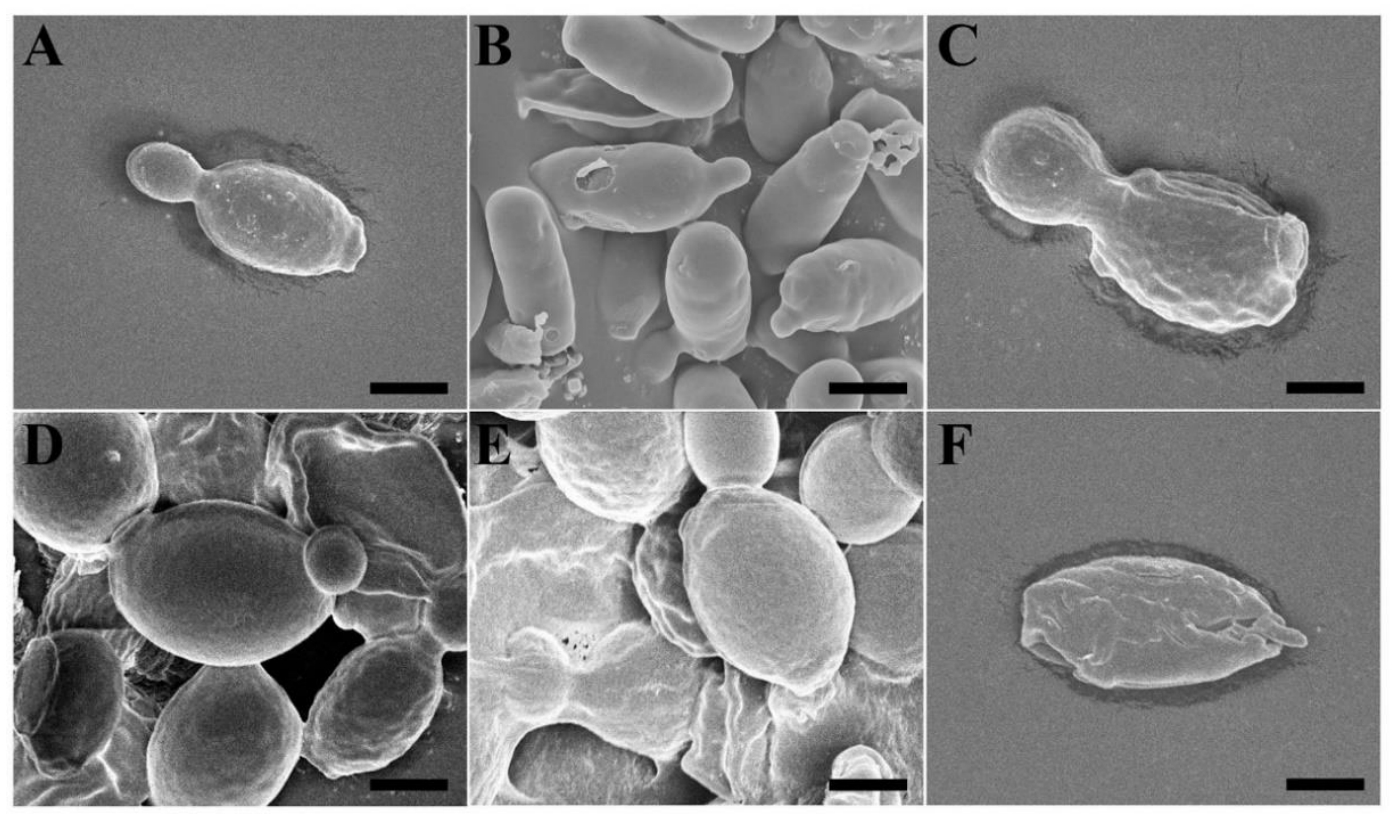

Figure 7. Time-dependent morphological changes in Vip3Aa-treated C. albicans cells. (A): control, (B): melittin ( $31.3 \mu \mathrm{g} / \mathrm{mL}$, $4 \mathrm{~h}$ incubation), (C): Vip3Aa $(62.5 \mu \mathrm{g} / \mathrm{mL}, 4 \mathrm{~h})$, (D): Vip3Aa $(62.5 \mu \mathrm{g} / \mathrm{mL}, 12 \mathrm{~h})$, (E): Vip3Aa $(62.5 \mu \mathrm{g} / \mathrm{mL}, 24 \mathrm{~h})$, and (F): Vip3Aa $(62.5 \mu \mathrm{g} / \mathrm{mL}, 48 \mathrm{~h})$. Bars represent $1 \mu \mathrm{m}$.

The main mechanism of antimicrobial peptides is to disrupt the cell membrane via pore formation and permeabilization, leading to rapid death. Because these mechanisms are different from those of conventional antibiotics, many researchers are interested in them as a next-generation antibiotic for antibiotic-resistant bacteria. Most antimicrobial proteins that have relatively large molecular weights penetrate into cells and exhibit antimicrobial actions via ROS generation and inhibition of cell metabolism. However, as shown in the antifungal action of Vip3Aa, it is extremely rare that the cells become ring-shaped and enlarged in size in cell phenotype. Although we suggest mitochondrial ROS generation and intracellular protein aggregation as an antifungal action of VIP3Aa in this study, further studies are needed to define multimodal actions. Studying the antimicrobial action of a protein with a large molecular weight can elucidate the intrinsic function of the protein in each organism, and it is also able to search for a new concept of antibiotic by studying its mechanism. In addition, if we find a domain or motif with antimicrobial effects in the protein sequence, an effective antimicrobial peptide can be designed.

\section{Materials and Methods}

\subsection{Materials}

Annexin V-FITC (Cat. No. A13199), MitoSOX Red (Cat. No. M36008), PI (CAS No. 25535-16-4), 5/6-carboxy-tetramethyl-rhodamine succinimidyl ester (NHS-rhodamine, CAS No. 246256-50-8) were obtained from Thermo Scientific (Waltham, MA, USA). All other reagents were of analytical grade.

\subsection{Fungal Cells}

The fungal cultures were sourced from the Korea Collection for Type Cultures (KCTC, Jeongup-si, Jeollabuk-do, Korea) and Culture Collection of Antimicrobial Resistant Microbes (CCARM, Seoul Women's University, Seoul, Korea). C. albicans (KCTC 7270), C. krusei (CCARM 14017), C. parapsilosis (CCARM 14016), C. tropicalis (KCTC 7221), Colletotrichum gloeosporioides (KCTC 6169), Fusarium graminearum (KCTC 16656), and F. solani (KCTC 6326) were obtained from KCTC or CCARM. 


\subsection{Cloning and Protein Expression of the Vip3Aa Gene in E. coli}

Polymerase chain reaction was used to amplify the Vip3Aa gene from a B. thuringiensis cDNA library. The recombinant protein Vip3Aa was expressed in E. coli strain BL21(DE3) after being cloned into the pET28a vector. The transformants in BL21 (DE3) cells were cultured at $37^{\circ} \mathrm{C}$ in LB medium, and the protein was induced by the addition of $0.4 \mathrm{mM}$ isopropyl-B-D-thiogalacto-pyranoside (IPTG) for $4 \mathrm{~h}$. The His-tagged Vip3Aa protein was purified on a Ni-NTA affinity column and fast protein liquid chromatography (FPLC) using a Superdex ${ }^{\circledR} 200$ Increase 10/300 GL column (GE healthcare, Waltham, MA, USA). SDS-PAGE analysis was used to identify intact Vip3Aa protein.

\subsection{Purification and Structural Analysis of the Vip3Aa Protein}

An FPLC (BioLogic DuoFlow Medium-Pressure Chromatography Systems, Bio-Rad, Hercules, CA, USA) system was used to further purify the isolated recombinant Vip3Aa. SEC was performed using a Superdex 200 column on the FPLC system at a flow rate of $0.6 \mathrm{~mL} / \mathrm{min}$, using $50 \mathrm{mM}$ sodium borate buffer at $25^{\circ} \mathrm{C}(\mathrm{pH}$ 9.5). A $10 \%$ SDS-PAGE was used to determine the purity of the Vip3Aa protein. Isolated proteins were digested with trypsin and subjected to peptide mass fingerprint analysis using matrix-assisted laser desorption ionization-time of flight (Microflex LRF 20, Bruker Daltonics, Billerica, MA, USA) to identify proteins resolved on SDS-PAGE gel. MASCOT software (available online: http:/ / matrixscience.com; accessed on 15 December 2021) was used to identify the proteins. A transmission electron microscope was used to examine the morphological changes in the Vip3Aa protein purified on SEC. Fractionated proteins were applied to carbon-coated copper grids that had been glow-discharged into the air (Harrick Plasma, Ithaca, NY, USA) and were then negatively stained with $2 \%$ uranyl acetate. The grids were examined using a 200 kV FEI Tecnai 20 TEM. Images were captured with a Gatan CCD camera.

\subsection{Antifungal Assay}

Spores of mold fungi grown on potato dextrose (PD; Difco, Sparks, MD, USA) agar plates were collected with PD broth consisting of $0.08 \%$ Triton X-100. Yeast cells were subcultured overnight in yeast extract-peptone-dextrose (YPD; Difco) medium. Fungal cells were adjusted to $2 \times 10^{4}$ spores (cells) $/ \mathrm{mL}$ in phosphate-buffered saline (PBS) (pH 7.4), containing $20 \%$ culture medium added to two-fold serially diluted proteins in 96-well plates. After $24 \mathrm{~h}$ (for yeast) or $48 \mathrm{~h}$ (for mold) incubation at $28{ }^{\circ} \mathrm{C}$, cell growth was examined microscopically with an inverted light microscope. The inhibitory concentration $50\left(\mathrm{IC}_{50}\right)$ against each fungus was defined as the lowest concentration of a sample that inhibited 50\% visible growth. All assays were performed in triplicate [30-32].

\subsection{Cytotoxicity Assay}

Fresh rat blood collected into a sodium heparin-coated tube (BD Vacutainer, Oxford, UK) was centrifuged at $800 \times g$ for $10 \mathrm{~min}$ and washed in PBS until the supernatant was clear. Eight $\%(v / v$, final concentration) of erythrocytes were added in the serially diluted proteins with PBS. After $1 \mathrm{~h}$ incubation at $37^{\circ} \mathrm{C}$ with mild agitation, the samples were centrifuged at $800 \times g$ for $10 \mathrm{~min}$, followed by an absorbance measurement of the supernatant at $414 \mathrm{~nm}$. Each assay was performed in triplicate, and the hemolysis percentage was calculated using the following equation:

$\%$ hemolysis $=\left[\left(\mathrm{Abs}_{414}\right.\right.$ in the protein solution- $\mathrm{Abs}_{414}$ in PBS $) /\left(\mathrm{Abs}_{414}\right.$ in $0.1 \%$ Triton-X100-Abs 414 in PBS $\left.)\right] \times 100$

$100 \%$ hemolysis was obtained by the absorbance of $0.1 \%$ triton $\mathrm{X}-100$ treatment, and $0 \%$ hemolysis was consisted of rat erythrocytes alone in PBS.

In vitro cytotoxicity assay was performed using the 2,3-Bis-(2-Methoxy-4-Nitro-5Sulfophenyl)-2H-Tetrazolium-5-Carboxanilide (XTT) assay in HaCaT (human keratinocyte) cells. The cells were cultured in Dulbecco's modified Eagle medium (DMEM; ThermoFisher Scientific, Gibco, Waltman, MA, USA) supplemented with antibiotic-antimycotic (Ther- 
moFisher Scientific, Gibco) and 10\% fetal bovine serum (ThermoFisher Scientific, Gibco) at $37^{\circ} \mathrm{C}$ in a humidified chamber in an atmosphere containing $5 \% \mathrm{CO}_{2}$. The cells were seeded at $5 \times 10^{4}$ cells $/ \mathrm{mL}$ in a 96-well microtiter plate. After $24 \mathrm{~h}$ of incubation, the cells were treated with two-fold serial dilutions of proteins, followed by another $24 \mathrm{~h}$ of incubation. The plate added by an activated XTT solution was additionally incubated for $4 \mathrm{~h}$, and the absorbance of each well was measured at wavelengths of 480 and $650 \mathrm{~nm}$ using a microtiter SpectraMax M5 reader (Molecular Devices, Sunnyvale, CA, USA).

\subsection{Membrane Integrity Assay Using PI}

C. albicans cells were incubated with the indicated proteins at $\mathrm{IC}_{50}$, and cells were washed twice with PBS and stained with PI $(10 \mu \mathrm{g} / \mathrm{mL})$. After additional incubation at $4{ }^{\circ} \mathrm{C}$ for $30 \mathrm{~min}$, cells were washed twice with PBS to remove unbound dye, and a quantitative analysis was performed using an Attune NxT acoustic focusing cytometer (Thermo Fisher Scientific Co., Waltham, MA, USA).

\subsection{Analysis Using CLSM}

To observe the cellular distribution of proteins, fungal cells were analyzed using a CLSM. After incubation with rhodamine-labeled peptides at $28^{\circ} \mathrm{C}$ for the presented times, the washed C. albicans cells were spotted on a cover glass with the mounting solution ( $50 \%$ glycerol, $0.1 \%$ n-propyl-gallate) and observed using CLSM (Nikon A1, Nikon Instruments Inc., Tokyo, Japan).

\subsection{MitoSOX Levels}

After incubation of fungal cells treated with proteins at $\mathrm{IC}_{50}$ for $12 \mathrm{~h}, 5 \mu \mathrm{M}$ of MitoSOX Red probe in PBS was applied to cells, followed by incubation for $10 \mathrm{~min}$ at $28{ }^{\circ} \mathrm{C}$ in dark. The incubated cells were washed three times with PBS and were analyzed using a fluorescence microscope and flow cytometry (Ex. $530 \mathrm{~nm} / \mathrm{Em} .575 \mathrm{~nm}$ ).

\subsection{Apoptosis Measurement}

Vip3Aa $(31.3,62.5$, and $125 \mu \mathrm{g} / \mathrm{mL})$ proteins were incubated with $C$. albicans cells for $12 \mathrm{~h}$ at $28^{\circ} \mathrm{C}$, and the cells were washed twice with PBS. The cells were stained with PI and FITC-labeled Annexin-V according to manufacturer's instructions and analyzed using an Attune NxT acoustic focusing cytometer.

\subsection{Analysis Using SEM}

Proteins were incubated with pre-cultivated $C$. albicans at $\mathrm{IC}_{50}$ in the presented concentrations and times. Cells were fixed overnight with PBS containing $5 \%$ glutaraldehyde at $4{ }^{\circ} \mathrm{C}$. The fixed cells were dehydrated in graded ethanol and critical point-dried under $\mathrm{CO}_{2}$. The gold-coated samples were observed using a field emission SEM (JSM-7100F, JEOL Ltd., Tokyo, Japan).

\section{Conclusions}

In summary, we discovered that Vip3Aa has a variety of antifungal effects by $\mathrm{pH}-$, concentration-, and time-dependent structural changes. During the growth of $C$. albicans cells, the intracellular $\mathrm{pH}$ varies between 6 and 7, owing to glucose metabolism. The important factor in the antifungal mechanism of Vip3Aa may be its structural variations in the cellular $\mathrm{pH}$. Accordingly, it is difficult to explain the antifungal effect of Vip3Aa with one mechanism. At the initial stage of treatment, it can attach to the cell wall and penetrate the cytosol. The penetrating Vip3Aa can be aggregated in acidic cytosol, suggesting it interferes with the movement of ions and substances into the cell. Morphological defects containing a ring-shape, increased size, and bust may relate to cell wall damaging action. Another mode of antifungal action is proposed, whereby Vip3Aa induces apoptosis of fungal cells via mitochondrial ROS production. This protein may play an important role in the Bacillus defense system against attack by fungal pathogens. Although the MIC value 
of Vip3Aa is relatively higher than that of other antimicrobial peptides, a synergistic effect will be achieved or antibiotic resistance will be overcome when it is treated in combination with antifungal agents having a different mechanism. However, further research is needed to establish its use in agricultural and clinical applications.

Author Contributions: Conceptualization, S.-C.P., M.-K.J., and J.R.L.; Methodology, S.-C.P., J.-Y.K., J.-K.L., and J.R.L.; Software, S.-C.P.; Validation, S.-C.P.; Formal analysis, S.-C.P., M.-K.J., and J.R.L.; Investigation, S.-C.P., J.-Y.K., J.-K.L., H.S.L., H.S., S.-H.Y., and S.-E.M.; Resources, S.-C.P.; Data curation, S.-C.P., J.-Y.K., J.-K.L., and J.R.L.; Writing-original draft preparation, S.-C.P.; Writing-review and editing, M.-K.J. and J.R.L.; Visualization, S.-C.P. and J.R.L.; Supervision, M.-K.J. and J.R.L.; Project administration, J.R.L.; Funding acquisition, J.R.L. All authors have read and agreed to the published version of the manuscript.

Funding: This research was supported by a grant from the National Institute of Ecology (NIE), funded by the Ministry of Environment (MOE) of the Republic of Korea (NIE-A-2021-07).

Institutional Review Board Statement: Not applicable.

Informed Consent Statement: Not applicable.

Data Availability Statement: Not applicable.

Conflicts of Interest: The authors declare no conflict of interest.

\section{References}

1. Dean, D.H. Biochemical genetics of the bacterial insect-control agent Bacillus thuringiensis: Basic principles and prospects for genetic engineering. Biotechnol. Genet. Eng. Rev. 1984, 2, 341-363. [CrossRef] [PubMed]

2. Liliana, P.L.; Mario, S.; Alejandra, A. Bacillus thuringiensis insecticidal three-domain Cry toxins: Mode of action, insect resistance and consequences for crop protection. FEMS Microbiol. Rev. 2012, 37, 3-22.

3. Estruch, J.J.; Warren, G.W.; Mullins, M.A.; Nye, G.J.; Craig, J.A.; Koziel, M.G. Vip3A, a novel Bacillus thuringiensis vegetative insecticidal protein with a wide spectrum of activities against lepidopteran insects. Proc. Natl. Acad. Sci. USA 1996, 93, 5389-5394. [CrossRef] [PubMed]

4. Bhalla, R.; Dalal, M.; Panguluri, S.K.; Jagadish, B.; Mandaokar, A.D.; Singh, A.; Kumar, P.A. Isolation, characterization and expression of a novel vegetative insecticidal protein gene of Bacillus thuringiensis. FEMS Microbiol. Lett. 2005, $243,467-472$. [CrossRef] [PubMed]

5. Yu, C.G.; Mullins, M.A.; Warren, G.W.; Koziel, M.G.; Estruch, J.J. The Bacillus thuringiensis vegetative insecticidal protein Vip3A lyses midgut epithelium cells of susceptible insects. Appl. Environ. Microbiol. 1997, 63, 532-536. [CrossRef]

6. Warren, G.W. Vegetative insecticidal proteins: Novel proteins for control of corn pests. In Advances in Insect Control: Role of Transgenic Plants; Carozzi, N., Koziel, M., Eds.; Taylor \& Francis: London, UK, 1997; pp. 109-121.

7. $\quad$ Estruch, J.J.; Koziel, M.G.; Yu, C.G.; Desai, N.M.; Nye, G.J.; Warren, G.W. Plant Pest Control. Patent WO 9844137, 8 October 1998.

8. Gupta, M.; Kumar, H.; Kaur, S. Vegetative insecticidal protein (Vip): A potential contender from Bacillus thuringiensis for efficient management of various detrimental agricultural pests. Front. Microbiol. 2021, 12, 659736. [CrossRef]

9. Han, S.; Craig, A.J.; Putnam, C.; Carozzi, N.B.; Tainer, A.J. Evolution and mechanism from structures of an ADP-ribosylating toxin and NAD complex. Nat. Genet. 1999, 6, 932-936. [CrossRef]

10. Leuber, M.; Orlik, F.; Schiffler, B.; Sickmann, A.A.; Benz, R. Vegetative insecticidal protein (Vip1Ac) of Bacillus thuringiensis HD201: Evidence for oligomer and channel formation. Biochemistry 2006, 45, 283-288. [CrossRef]

11. Lee, M.K.; Walters, F.S.; Hart, H.; Palekar, N.; Chen, J.-S. The mode of action of the Bacillus thuringiensis vegetative insecticidal protein Vip3A differs from that of Cry1Ab $\delta$-Endotoxin. Appl. Environ. Microbiol. 2003, 69, 4648-4657. [CrossRef]

12. Dow, J. Extremely high $\mathrm{pH}$ in biological systems: A model for carbonate transport. Am. J. Physiol. Integr. Comp. Physiol. 1984, 246, R633-R636. [CrossRef]

13. Dow, J. pH gradients in lepidopteran midgut. J. Exp. Biol. 1992, 172, 355-375. [CrossRef] [PubMed]

14. Doss, V.A.; Kumar, K.A.; Jayakumar, R.; Sekar, V. Cloning and expression of the vegetative insecticidal protein (vip3V) gene of Bacillus thuringiensis in Escherichia coli. Protein Expr. Purif. 2002, 26, 82-88. [CrossRef]

15. Chen, J.; Yu, J.; Tang, L.; Tang, M.; Shi, Y.; Pang, Y. Comparison of the expression of Bacillus thuringiensis full-length and N-terminally truncated vip3A gene in Escherichia coli. J. Appl. Microbiol. 2003, 95, 310-316. [CrossRef] [PubMed]

16. Li, C.; Xu, N.; Huang, X.; Wang, W.; Cheng, J.; Wu, K.; Shen, Z. Bacillus thuringiensis Vip3 mutant proteins: Insecticidal activity and trypsin sensitivity. Biocontrol Sci. Technol. 2007, 17, 699-708. [CrossRef]

17. Selvapandiyan, A.; Arora, N.; Rajagopal, R.; Jalali, S.K.; Venkatesan, T.; Singh, S.P.; Bhatnagar, R.K. Toxicity analysis of N- and C-Terminus-deleted vegetative insecticidal protein from Bacillus thuringiensis. Appl. Environ. Microbiol. 2001, 67, 5855-5858. [CrossRef]

18. Bel, Y.; Banyuls, N.; Chakroun, M.; Escriche, B.; Ferré, J. Insights into the structure of the Vip3Aa insecticidal protein by protease digestion analysis. Toxins 2017, 9, 131. [CrossRef] 
19. Banyuls, N.; Hernández-Martínez, P.; Quan, Y.; Ferré, J. Artefactual band patterns by SDS-PAGE of the Vip3Af protein in the presence of proteases mask the extremely high stability of this protein. Int. J. Biol. Macromol. 2018, 120, 59-65. [CrossRef] [PubMed]

20. Zack, M.D.; Sopko, M.S.; Frey, M.L.; Wang, X.; Tan, S.Y.; Arruda, J.M.; Letherer, T.T.; Narva, K.E. Functional characterization of Vip3Ab1 and Vip3Bc1: Two novel insecticidal proteins with differential activity against lepidopteran pests. Sci. Rep. 2017, 7, 11112. [CrossRef]

21. Quan, Y.; Ferré, J. Quan structural domains of the Bacillus thuringiensis Vip3Af protein unraveled by Tryptic digestion of Alanine Mutants. Toxins 2019, 11,368. [CrossRef]

22. Shao, E.; Zhang, A.; Yan, Y.; Wang, Y.; Jia, X.; Sha, L.; Guan, X.; Wang, P.; Huang, Z. Oligomer formation and insecticidal activity of Bacillus thuringiensis Vip3Aa toxin. Toxins 2020, 12, 274. [CrossRef]

23. Chakroun, M.; Banyuls, N.; Bel, Y.; Escriche, B.; Ferré, J. Bacterial vegetative insecticidal proteins (Vip) from entomopathogenic bacteria. Microbiol. Mol. Biol. Rev. 2016, 80, 329-350. [CrossRef]

24. Palma, L.; Scott, D.J.; Harris, G.; Din, S.-U.; Williams, T.L.; Roberts, O.J.; Young, M.T.; Caballero, P.; Berry, C. The Vip3Ag4 insecticidal protoxin from Bacillus thuringiensis adopts a tetrameric configuration that is maintained on proteolysis. Toxins 2017, 9, 165. [CrossRef]

25. Park, S.C.; Kim, J.Y.; Shin, S.O.; Jeong, C.Y.; Kim, M.H.; Shin, S.Y.; Cheong, G.W.; Park, Y.; Hahm, K.S. Investigation of toroidal pore and oligomerization by melittin using Transmission electron mcroscopy. Biochem. Biophys. Res. Commun. 2004, 321, 631-637. [CrossRef]

26. Liao, C.; Selvan, M.E.; Zhao, J.; Slimovitch, J.L.; Schneebeli, S.; Shelley, M.; Shelley, J.C.; Li, J. Melittin aggregation in aqueous solutions: Insight from molecular dynamics simulations. J. Phys. Chem. B 2015, 119, 10390-10398. [CrossRef]

27. Yang, Z.; Zheng, J.; Chan, C.-F.; Wong, I.L.; Heater, B.S.; Chow, L.M.; Lee, M.M.; Chan, M.K. Targeted delivery of antimicrobial peptide by Cry protein crystal to treat intramacrophage infection. Biomaterials 2019, 217, 119286. [CrossRef] [PubMed]

28. Raheem, N.; Straus, S.K. Mechanisms of action for antimicrobial peptides with antibacterial and antibiofilm functions. Front. Microbiol. 2019, 10, 2866. [CrossRef] [PubMed]

29. Park, S.-C.; Park, Y.; Hahm, K.-S. The role of antimicrobial peptides in preventing multidrug-resistant bacterial infections and biofilm formation. Int. J. Mol. Sci. 2011, 12, 5971-5992. [CrossRef] [PubMed]

30. Park, S.-C.; Kim, H.; Kim, J.-Y.; Kim, H.; Cheong, G.-W.; Lee, J.R.; Jang, M.-K. Improved Cell Selectivity of Pseudin-2 via substitution in the Leucine-Zipper motif: In Vitro and In Vivo Antifungal Activity. Antibiotics 2020, 9, 921. [CrossRef]

31. Park, S.-C.; Kim, I.R.; Kim, J.-Y.; Lee, Y.; Yoo, S.-H.; Jung, J.H.; Cheong, G.-W.; Lee, S.Y.; Jang, M.-K.; Lee, J.R. Functional characterization of a rice Thioredoxin protein OsTrxm and its cysteine mutant variant with antifungal activity. Antioxidants 2019, 8, 598. [CrossRef]

32. Kim, J.-Y.; Park, S.-C.; Noh, G.; Kim, H.; Yoo, S.-H.; Kim, I.R.; Lee, J.R.; Jang, M.-K. Antifungal effect of a chimeric peptide Hn-Mc against pathogenic fungal strains. Antibiotics 2020, 9, 454. [CrossRef]

33. Chen, R.-C.; Lan, C.-Y. Human antimicrobial peptide hepcidin 25-induced Apoptosis in Candida albicans. Microorganism 2020, 8, 585. [CrossRef] [PubMed]

34. Choi, H.; Lee, D.G. The influence of the N-terminal region of antimicrobial peptide pleurocidin on fungal apoptosis. J. Microbiol. Biotechnol. 2013, 23, 1386-1394. [CrossRef] [PubMed]

35. Dantas, A.D.S.; Day, A.; Ikeh, M.; Kos, I.; Achan, B.; Quinn, J. Oxidative stress responses in the human fungal pathogen, Candida albicans. Biomolecules 2015, 5, 142-165. [CrossRef] [PubMed]

36. Scherz-Shouval, R.; Elazar, Z. ROS, mitochondria and the regulation of autophagy. Trends Cell Biol. 2007, 17, 422-427. [CrossRef] [PubMed] 